\title{
N400-like Magnetoencephalography Responses Modulated by Semantic Context, Word Frequency, and Lexical Class in Sentences
}

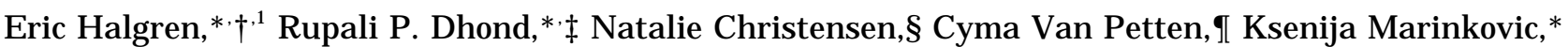 \\ J effrey D. Lewine,§ and Anders M. Dale* \\ *MGH-NMR Center, Harvard Medical School, Boston, Massachusetts; †I NSERM E9926, Marseilles, France; §Department of Radiology \\ and $\ddagger$ Neuroscience Program, University of Utah, Salt Lake City, Utah; and \\ IDepartment of Psychology, University of Arizona, Tucson, Arizona
}

Received February 25, 2002

Words have been found to elicit a negative potential at the scalp peaking at $\sim \mathbf{4 0 0}$ ms that is strongly modulated by semantic context. The current study used whole-head magnetoencephalography (MEG) as male subjects read sentences ending with semantically congruous or incongruous words. Compared with congruous words, sentence-terminal incongruous words consistently evoked a large magnetic field over the left hemisphere, peaking at $\sim \mathbf{4 5 0} \mathrm{ms}$. Source modeling at this latency with conventional equivalent current dipoles (ECDs) placed the $\mathrm{N} 400 \mathrm{~m}$ generator in or near the left superior temporal sulcus. A distributed solution constrained to the cortical surface suggested a sequence of differential activation, beginning in Wernicke's area at $\sim 250 \mathrm{~ms}$, spreading to anterior temporal sites at $\sim 270 \mathrm{~ms}$, to Broca's area by $\sim 300 \mathrm{~ms}$, to dorsolateral prefrontal cortices by $\sim 320 \mathrm{~ms}$, and to anterior orbital and frontopolar cortices by $\sim 370$ ms. Differential activity was exclusively left-sided until $>\mathbf{3 7 0} \mathrm{ms}$, and then involved right anterior temporal and orbital cortices. At the peak of the $\mathbf{N} 400 \mathrm{~m}$, activation in the left hemisphere was estimated to be widespread in the anterior temporal, perisylvian, orbital, frontopolar, and dorsolateral prefrontal cortices. In the right hemisphere, the orbital, as well as, weakly, the right anterior temporal cortices were activated. Similar but weaker field patterns were evoked by intermediate words in the sentences, especially to low-frequency words occurring in early sentence positions where there is little preceding context. The locations of the $\mathbf{N} 400 \mathrm{~m}$ sources identified with the distributed solution correspond well with those previously demonstrated with direct intracranial recordings, and suggested by functional magnetic resonance imaging (fMRI). These results help identify a distributed cortical network that supports online semantic processing. 2002 Elsevier Science (USA)

\footnotetext{
${ }^{1}$ To whom correspondence should be addressed at Massachusetts General Hospital Nuclear Magnetic Resonance Center, Room 2301, Building 149, 13th Street, Charlestown, MA 02129. Fax: (617) 7267422. E-mail: halgren@nmr.mgh.harvard.edu.
}

Key Words: reading; sentences; semantic context; lexicon; magnetoencephalography; temporal lobe; prefrontal cortex; language; perirhinal cortex.

\section{INTRODUCTION}

The N400 was first described as a negative-going component in the event-related potential (ERP), larger in amplitude for semantically anomalous sentence completions (Kutas and Hillyard, 1980). F or example, the sentence "Mary was hungry so she went to the store to buy some food" elicits a small N400, whereas "Mary was hungry so she went to the store to buy some chairs" elicits a large N400, with the difference beginning at $\sim 240 \mathrm{~ms}$ post stimulus onset and peaking at $\sim 400 \mathrm{~ms}$. This semantic context effect is not restricted to comparisons between congruous and anomalous words. When pairs or lists of words are presented visually, the amplitude of the N400 is smaller if the eliciting word is semantically related rather than unrelated to the preceding word. For words in sentences, N400 amplitude is determined by the degree of contextual constraint imposed by the preceding portion of the sentence; highly predictable final words elicit smaller $\mathrm{N} 400$ s than congruent but unlikely words, which in turn elicit smaller N400s than completely anomalous final words. Sentence-intermediate words elicit N400s of graded amplitude depending on their position within the sentence. In a list of unrelated sentences (as opposed to connected discourse) early words elicit larger $\mathrm{N} 400$ s than later words as the later words can benefit from a larger amount of preceding context (for reviews see Kutas and Van Petten, 1994; Van Petten, 1995; Kutas and Federmeier, 2000). Auditory words and signs in American Sign Language yield N400 semantic context effects similar to those observed for printed words (Neville et al., 1997; Van Petten et al., 1999). This scalp potential is thus a robust index of the ease or difficulty of semantic processing. 


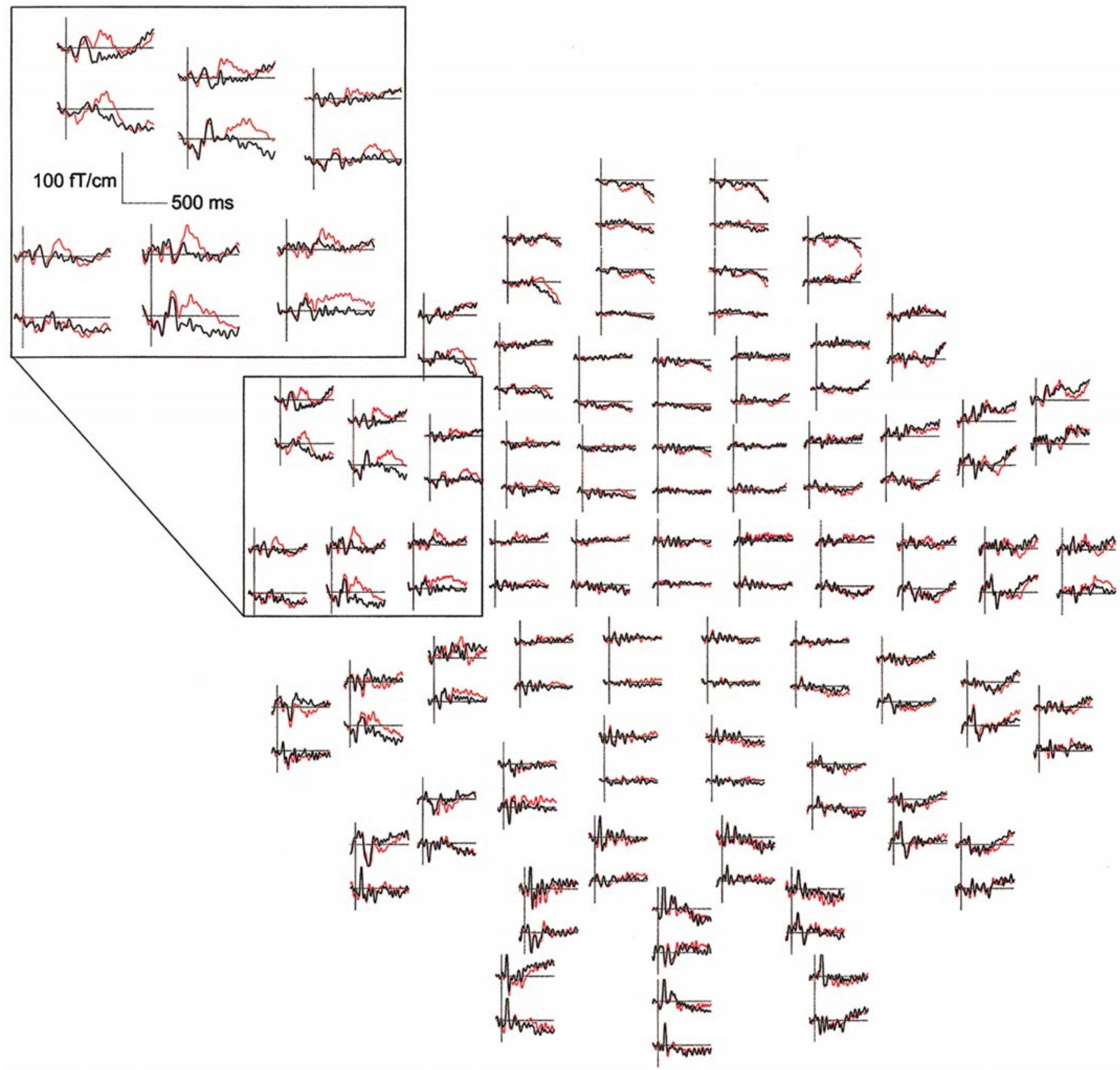

FIG. 1. MEG responses evoked by congruous (red) and incongruous (black) sentence-terminal words in subject 1 . Waveforms from 122 gradiometer channels are shown. The major difference between conditions peaks at $\sim 400 \mathrm{~ms}$ in the anterior temporal region. Signals from six pairs of gradiometers are shown in expanded format at the upper left.

N400 context effects have also been observed for nonverbal but meaningful stimuli, albeit with somewhat different scalp distributions than for words. When line drawings are preceded by either printed sentences or other line drawings, the difference between semantically related and unrelated drawings is similar to the linguistic N400 in waveshape and latency, but somewhat larger at anterior scalp sites ( $\mathrm{Hol}$ comb and McPherson, 1994; Ganis et al., 1996). Inter- pretable environmental sounds (glass breaking, horse hooves on pavement) also elicit N400-like potentials that are modulated by semantic context, but the context effect for these nonspeech sounds has a hemispheric asymmetry different from that observed for words (Van Petten and Rheinfel der, 1995; Plante et al., 2000). In contrast to these results from various sorts of stimuli, which convey a meaning (or could possibly convey a meaning, as in the case of pronounceable 

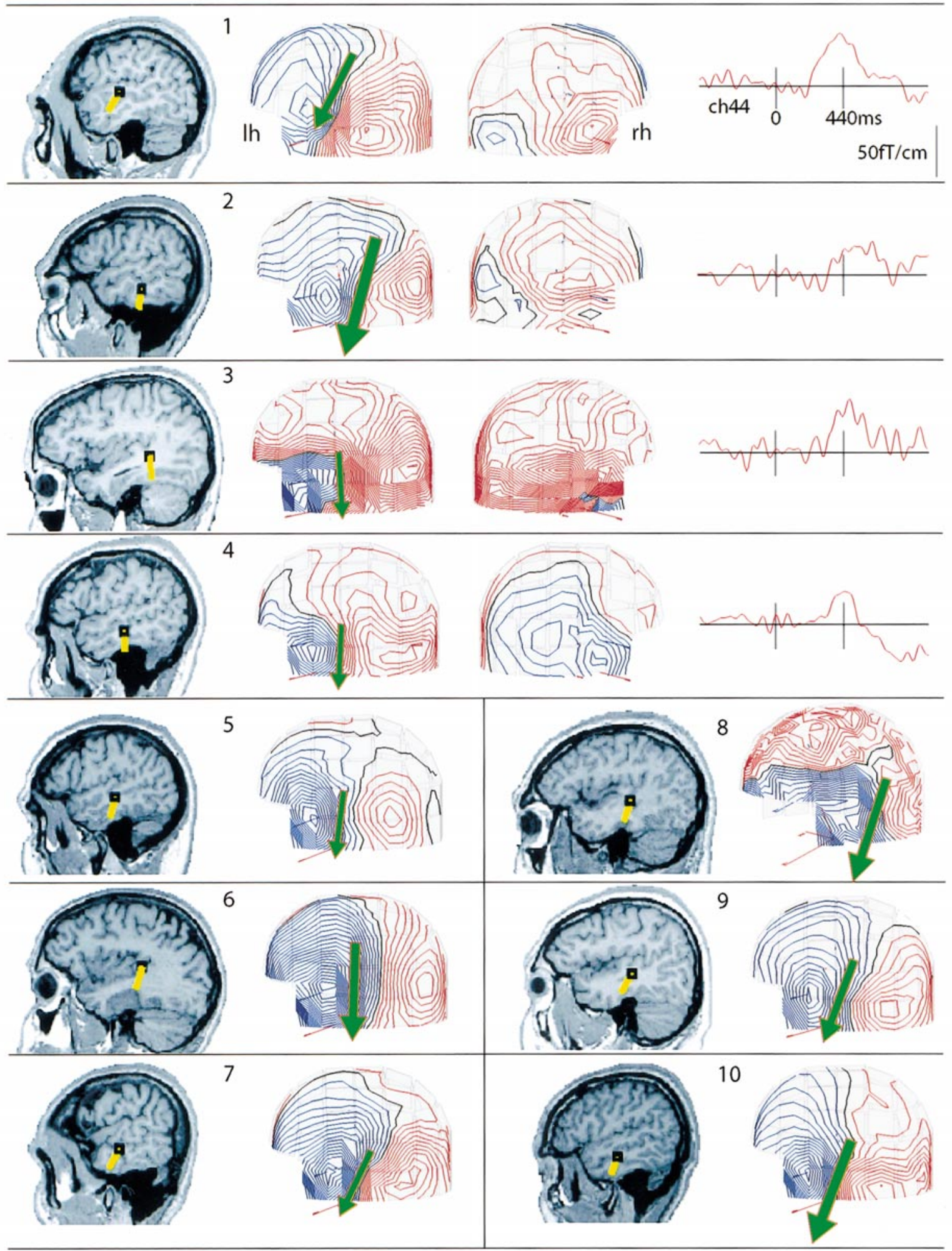

FIG. 2. ECD localization, $M E G$ field maps, and time courses to incongruous-minus-congruous words. ECDs on sagittal $M R$ images are consistently localized in or near the superior temporal sulcus in all 10 subjects. In the next column, MEG fields at the peak response latency of $\sim 400 \mathrm{~ms}$ are illustrated as contour maps with the step between lines set at $20 \mathrm{fT}$, and current entering the brain as blue. Dipolar field patterns are most apparent over the left hemisphere and are consistent across individual subjects. Fields over both hemispheres and the MEG signal in sensor 44 are shown for the first four subjects. 
nonwords), stimuli that cannot be mapped onto existing semantic knowledge (novel geometric shapes, unpronounceable nonwords, etc.) do not elicit N400s (Smith and Halgren, 1987; Van Petten and Senkfor, 1996). The differing scal p distributions of the family of N400 potentials elicited by words versus nonverbal stimuli suggest the engagement of different (although likely overlapping) populations of neurons in processing their meanings. Overall, N400-like potentials seem to be triggered by stimuli whose physical forms can be used to access other knowledge about the referent of the stimulus (Halgren, 1990).

Even for the N400 elicited by words, little progress has been made in inferring the underlying brain generators from the EEG scalp distribution of the component. The word-elicited N400 is largest at midline centroparietal scalp sites, and larger at lateral sites over the right hemisphere as compared with the left (Kutas and Hillyard, 1982; Kutas et al., 1988b; Van Petten and Rheinfelder, 1995). This right-greater-than-left scalp asymmetry is a clear indication of the difficulty of mapping scalp distribution onto cortical regions, as studies of both split-brain patients and patients with unilateral damage have suggested that the N400 context effect is critically dependent on an intact left hemisphere (Kutas et al., 1988a; Hagoort et al., 1996; Friederici et al., 1998), as is the $\mathrm{N} 400$ repetition effect (Smith and Halgren, 1989). High-density EEG recordings were unable to identify topographic features of the N400 using Laplacian estimates of radial current density (Curran et al., 1993). Similarly, minimum-norm and minimum-current distributed solutions constrained to lie on the cortical surface reconstructed from MRI produced no findings that were consistent across individuals (Haan et al., 2000). Furthermore, since the same scal p topography can result from different intracranial generators (Dale and Halgren, 2001), it is possible that the $\mathrm{N} 400$ s evoked by different stimuli in different tasks are actually arising in substantially different regions.

The aim of the current study is to attempt to localize the N400 in normal subjects using event-related magnetic fiel ds (by magnetoencephalography, MEG). While MEG fields and ERPs are both generated by cortical transsynaptic current flows, they are differentially sensitive to activity in different cortical locations depending on the local orientation and depth of active synapses (Hamalainen et al., 1993). Furthermore, MEG is less sensitive than EEG to the effects of intervening tissues on propagation of the activity from the generator to the sensor.

To establish a clear relationship between the MEG field sources and the many studies of language processing using ERPs, we examined multiple aspects of word and sentence processing, all of which have been reported to modulate the amplitude of the electrical N400. Two manipulations of semantic context have already been described: a comparison between congruent and semantically anomalous sentence-terminal words, and a subtler comparison between content words (nouns, verbs, adjectives, and -ly adverbs) occurring in early versus late sentence-intermediate positions. Sentence-intermediate words were also split according to frequency of use in English, as less commonly used words (e.g., "twins," "cartoon," "vase") elicit larger N400s than high-frequency words (e.g., "parents," "patient," "time") (Smith and Halgren, 1987). In electrical recordings, this word frequency effect interacts with word order, such that high- and low-frequency words elicit equivalent N400s in late word orders, after some semantic context has accrued (Van Petten and Kutas, 1990, 1991; Van Petten and Rheinfelder, 1995). Finally, we also compare content words with function words (e.g., determiners, pronouns, conjunctions, prepositions) because function words elicit smaller electrical N400s (Kutas and Hillyard, 1983; Van Petten and Kutas, 1991; Munte et al., 2001).

In contrast to the lack of clear localization from scalp recordings, direct intracranial recordings have convincingly localized cortical activity in a variety of paradigms, which modulate scalp-recorded N400 amplitude. These include comparisons between congruous and incongruous sentence completions (McCarthy et al., 1995), related and unrelated word pairs (Nobre et al., 1994; Nobre and McCarthy, 1995), high- and lowfrequency words (Fernandez et al., 1998), content and function words (Nobre and McCarthy, 1995), and repeated versus initial presentations of isolated words (Smith et al., 1986; Puce et al., 1991; Halgren et al., 1994a,b; Elger et al., 1997; Helmstaedter et al., 1997; Fernandez et al., 1998; Grunwald et al., 1998). Many of these studies have converged to suggest an N400 generator in the anterior medial temporal lobe (AMTL). In addition to the $A M T L$, intracranial recordings have demonstrated prominent N400 generators in the posteroventral prefrontal cortex (Halgren et al., 1994a; Guillem et al., 1999; Marinkovic et al., 2000), Iateral temporal lobe in the vicinity of the superior temporal sulcus (Halgren et al., 1994b; Guillem et al., 1995; Elger et al., 1997), and posterior parietal sites (Halgren et al., 1994b; Guillem et al., 1999).

Although the intracranial data identify candidategenerating structures, their propagation to the scalp is difficult to determine. Further complicating the use of such data to specify the neural sources of the scalprecorded $\mathrm{N} 400$ is that they are obtained from electrodes implanted in patients with long-standing epilepsy, especially in seizure-prone regions, and thus may be subject to contamination from the pathology. Furthermore, few of the intracranial studies have included more than one experimental contrast. Many of the manipulations above influence other components of the scalp-recorded ERP in addition to the N400. Word 
repetition, for instance, modulates the amplitude of both the N400 and a later, but temporally overlapping scalp positivity. These two scalp repetition effects frequently co-occur in the normal population, but are dissociated in amnesic subjects with normal language comprehension (Olichney et al., 2000), so that it is important to differentiate their neural generators as well (for reports of multiple repetition-sensitive components in intracranial data see Smith et al., 1986; Halgren et al., 1994b; Elger et al., 1997). Similarly, content and function words are differentiated by scalp components recorded over frontal cortex in addition to the centroparietal N400 (Kutas and Van Petten, 1994; King and Kutas, 1998; Munte et al., 2001). Overall, we believe that a convergent-measures approach of including several N400 manipulations is most likely to clarify the relationship between scalp-recorded data and their neural sources.

A second innovation of the current study is the application of a distributed source modeling procedure to the MEG results. Previous studies using MEG have localized an equivalent current dipole (ECD) for the $\mathrm{N} 400 \mathrm{~m}$ to the left posterosuperior temporal lobe (Simos et al., 1997; Helenius et al., 1999). However, dipole modeling methods work best when the generator is a single focal source. When extensive multiple generators are active, distributed source estimation procedures may be more realistic (Dale and Halgren, 2001). Since the intracranial studies reviewed above indicate that the N400 may be associated with multiple extensive generators, the current study modeled N400 generation using a distributed source modeling procedure, as well as ECDs to allow comparison with earlier studies.

Our results suggest that the $\mathrm{N} 400 \mathrm{~m}$ is sensitive to the same psycholinguistic factors influencing the electrical N400 ("N400e"), and further that a distributed model of the N400m corresponds well with the intracranial results. Additionally, the model suggests that the underlying process is spatiotemporally dynamic, spreading over a period of $250 \mathrm{~ms}$ from Wernicke's area to more anterior left hemisphere sites and then to the right hemisphere.

\section{METHODS}

\section{Participants and Stimuli}

Ten healthy right-handed men (aged 20-26) served as volunteers. Subjects with a history of head injury, neurological or psychiatric disease, or substance abuse were excluded. Subjects were screened to eliminate those with frequent blinking or MEG artifacts (e.g., due to dental work). All were experienced in MEG experiments and selected on the basis of reliability and compliance with instructions.
Each participant read 240 sentences presented one word at a time (7-29 words per sentence) on a monitor controlled by a Maclntosh computer (MacProbeTM software (Hunt, 1994)). Word duration was $200 \mathrm{~ms}$ per word followed by a 133-ms interword interval. Twelve hundred milliseconds after the onset of the sentenceterminal word, the subject was cued to blink twice at 1-s intervals. A fixation target $(\ll * \gg)$ was then presented for $200 \mathrm{~ms}$, followed after $800 \mathrm{~ms}$ by the next sentence. A 15-s rest was inserted every 40 sentences. Words were presented as white letters on a black background, and subtended a visual angle of $\sim 1.5^{\circ}$ vertically and less than $7.5^{\circ}$ horizontally. Participants were instructed to read for comprehension, and warned that they would be tested for memory following the recording session.

Half of the sentences ended with a predictable, semantically congruent word (cloze probability of greater than $70 \%$ in a different group of college undergraduates); half ended in a semantically incongruent word. Congruous and incongruous final words were matched for word frequency and length in letters. Sentences with congruous and incongruous endings were randomly intermixed.

Subsets of words occurring in sentence-intermediate positions were coded as Early content words; these were the first open-class words of their sentences and occurred in position 2, 3, or 4. Late content words occurred in word orders 5 through 12, but never included sentence-terminal words. Early and Late words were further subdivided by frequency of usage as high frequency, i.e., with the sum of all regularly inflected versions, $>30$ per million in the Kucera and Francis (1967) corpus, mean frequency of 336, or low frequency $(<30$, mean frequency of 15$)$. When collapsed across frequency, the sets of Early and Late words were equivalent in frequency and word length, as seen in Table 1. Similarly, when collapsed across word order and high and low-frequency, words were equivalent in word order and length. Finally, all content words were compared with function words, which were both shorter in length $(P<0.001)$ and more frequent in usage $(P<$ 0.001).

\section{Recording Procedures}

MEG signals were recorded from 122 channels at 0.03-100 Hz using a Neuromag-122 instrument (4D Neuroimaging, San Diego, CA) with orthogonal pairs of planar gradiometers at each of 61 locations over the entire scalp (Ahonen et al., 1993; Hamalainen et al., 1993). Trials from different conditions were averaged for each subject after rejecting trials with eyeblinks or other artifacts using amplitude criteria confirmed with visual inspection.

In a separate imaging session separated by at least $24 \mathrm{~h}$ from the MEG experiment, high-resolution 3D 


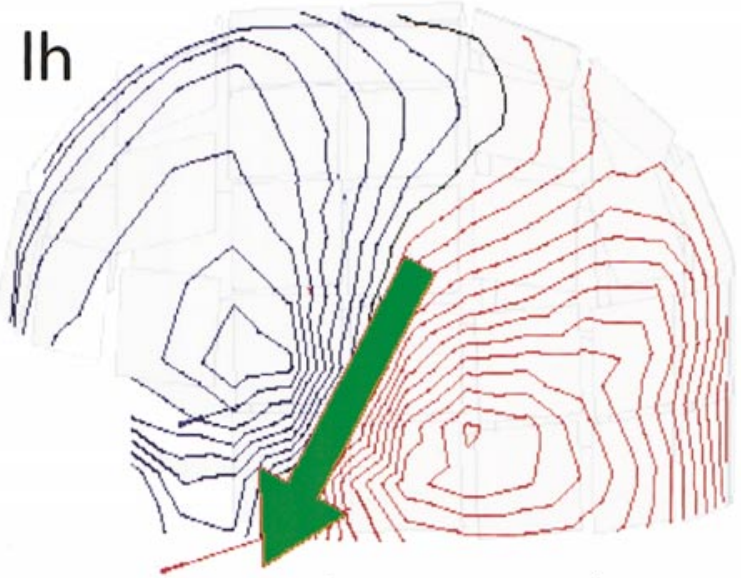

\section{semantic congruity incongruent-congruent}

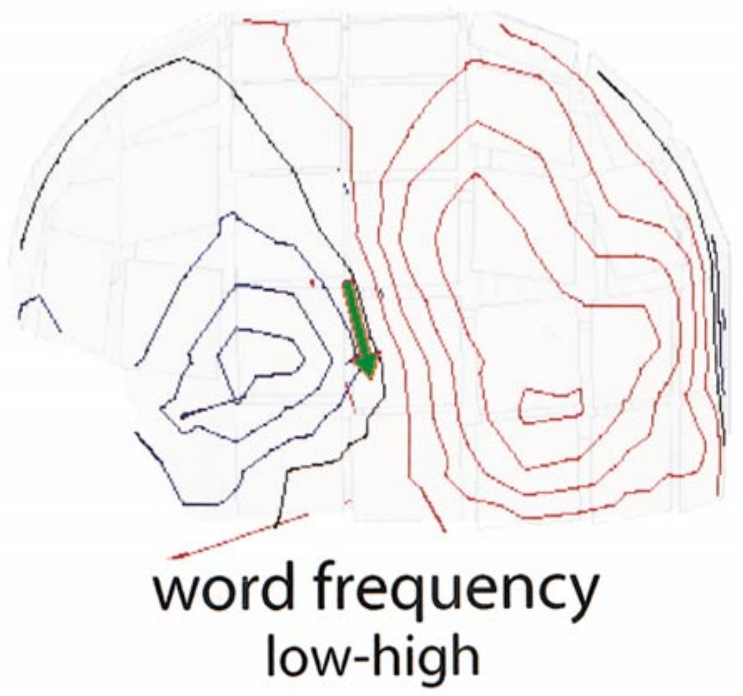

3

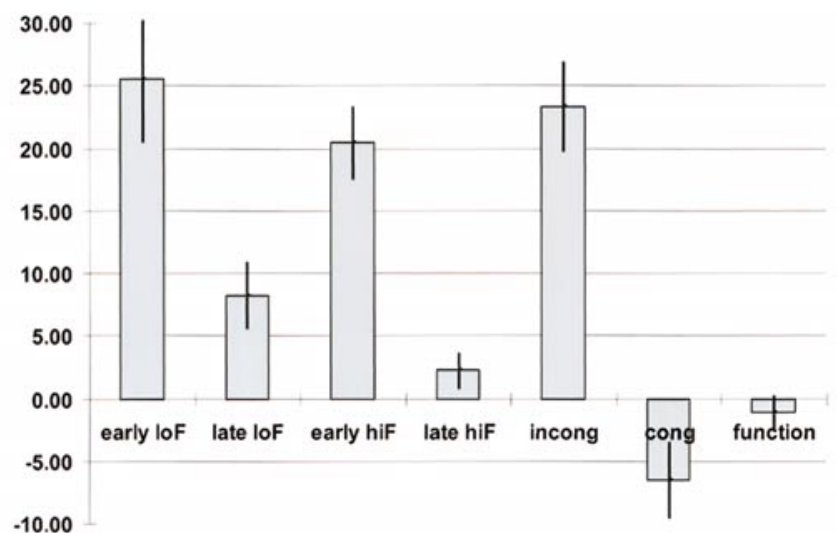

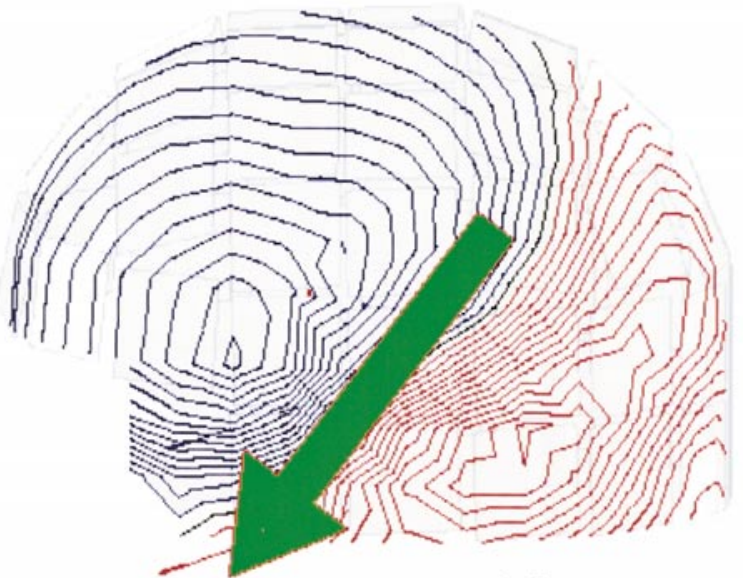

\section{sentence position early-late}

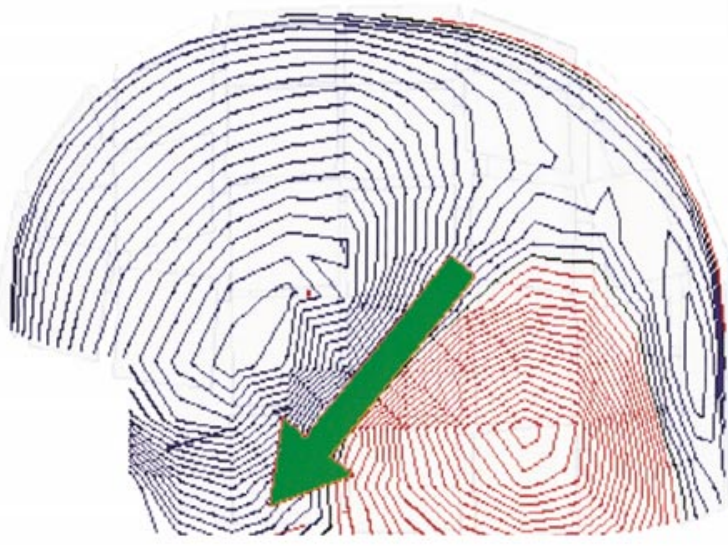

\section{lexical class content-function}

FIG. 3. MEG field differences associated with different word characteristics in subject 1. Fields are illustrated as contour maps with the step between lines set at $20 \mathrm{fT}$. ECDs modeled on the individual fields are superimposed on the contour maps, at the peak latency near 400 ms. Similar patterns are associated with differences in semantic congruity for sentence-terminal words, sentence position (words occurring early vs late in the sentence), and lexical class (intermediate words in the sentence that either convey content or function to provide syntactic linkage). Word frequency (intermediate words that occur seldom or often in common usage) has a smaller effect. 


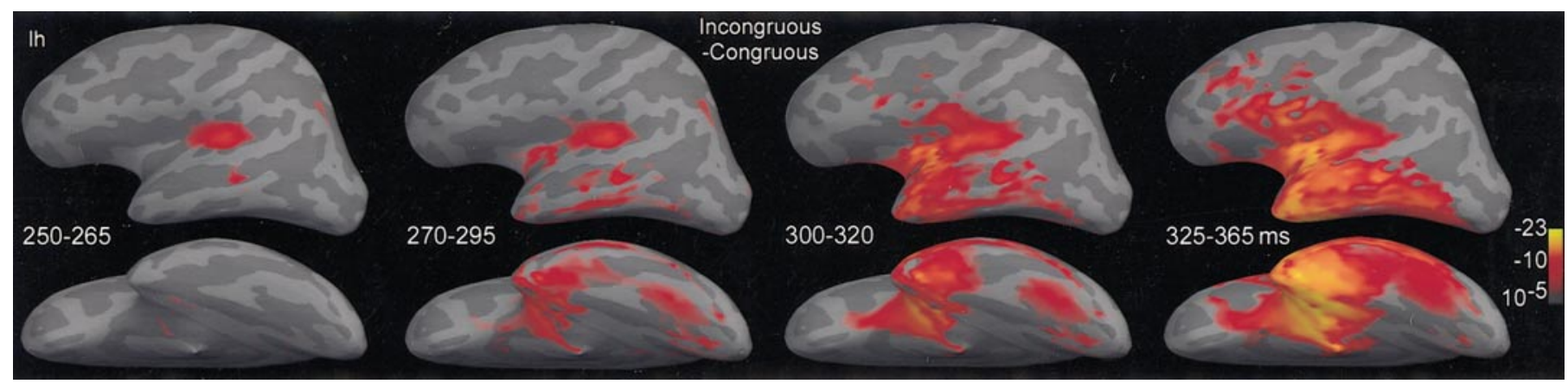

FIG. 6. Averaged cortical activity patterns to sentence-terminal incongruous minus congruous words in four latency windows prior to 400 ms. Activation is exclusively left hemisphere. It is estimated to begin in Wernicke's area at $\sim 250$ ms, spreads to anterior temporal sites at $\sim 270 \mathrm{~ms}$, to the prefrontal cortex by $\sim 300 \mathrm{~ms}$, and becomes increasingly widespread by $\sim 325 \mathrm{~ms}$. Activation is averaged across subjects and shown as significance levels on the average inflated cortical surface. Threshold $\mathrm{P}<10^{-5}$; full red $\mathrm{P}<10^{-10}$; full yellow $\mathrm{P}<10^{-23}$.

T1-weighted magnetic resonance (MR) images were acquired for each subject using a 1.5-T Picker Eclipse (Marconi Medical, Cleveland, OH). The MEG sensor coordinate system was aligned with the MRI coordinate system using three head position ( $\mathrm{HPI}$ ) coils, attached to the scalp (Hamalainen et al., 1993). The HPI coils generate weak magnetic signals, and thus can be directly localized by the magnetoencephalograph sensors. The positions of the HPI coils with respect to the subject's head (and thus MR image) were determined by measuring multiple points (including the HPI coils) using a Polhemus FastTrack 3D digitizer. Multiple measurements of the HPI coils were used to monitor for head movement, which was minimized using a chinstrap and foam padding on the sides of the head.

\section{Equivalent Current Dipole Modeling}

The rationale and procedure for ECD source modeling used here have been extensively described (Hamalainen et al., 1993). Briefly, the locations, orientations, and strengths of the ECDs were found with a least-squares fit in a spherical volume conductor, the center of which was equal to the local center of curvature of the surface of the brain in the occipital-parietal area, as determined on the individual MR images.

Analysis was terminated 400 ms after stimulus onset for comparisons between sentence-intermediate content and function words. Sentence structure is such that, for any given word type, content words tend to be followed by function words, and vice versa. In our sentences, $65 \%$ of the intermediate content words are fol- lowed by function words, and $60 \%$ of function words are followed by content words. Thus, late differences in brain activity to the two word types could be due to differences in the subsequent words. The problem of subsequent word overlap is especially problematic in comparing content and function words because it is confounded with their differences in word length which would also be expected to influence short-latency activity. Note that with the stimulus-onset asynchrony of 333 ms used in this study, and assuming that activation requires $\sim 70 \mathrm{~ms}$ to reach the cortex, cortical processing of the word following the word of interest would commence $\sim 403 \mathrm{~ms}$ after stimulus onset. Consequently, to avoid contamination with activity evoked by the subsequent word, only latencies prior to $400 \mathrm{~ms}$ were examined in these analyses.

A slightly less conservative analysis endpoint, 450 ms, was chosen for comparisons between sentence-intermediate content words differing in frequency or position. This is $\sim 117 \mathrm{~ms}$ after the first cortical activation, and probably prior to the arrival of activation in the temporofrontal areas of interest in this analysis. F urthermore, because both conditions in these comparisons are content words, there is no obvious difference in the characteristics of the subsequent word.

\section{Distributed Solutions}

Cortical surface reconstruction. Geometrical representations of the cortical surface were constructed from the structural MR image using procedures described previously (Dale and Sereno, 1993; Dale et al., 1999;

FIG. 4. Average activation to different word types. N400m activity was estimated in 10 subjects from 360 to 450 ms to different word types using the ECDs shown in Fig. 3. The average activation was greatest for words occurring early in the sentence; word frequency had a smaller effect that was not significant. Sentence-terminal incongruous words evoked a response about equal to that to early words. No discernable response was present to function words.

FIG. 5. Estimated cortical activity patterns in subject 1 to sentence-terminal incongruous minus congruous words. Activation is shown as significance levels on the subject's cortical surface, after it has been inflated to reveal the sulcal cortex (darker gray areas). Activation is most significant in the left anterior temporal and posteroventral prefrontal cortices. Threshold $\mathrm{P}<10^{-5}$; full red $\mathrm{P}<10^{-10}$; full yellow $\mathrm{P}<$ $10^{-23}$. 
TABLE 1

Characteristics of Word Stimuli

\begin{tabular}{lccrr}
\hline \multicolumn{1}{c}{ Word category } & Order in sentence & No. of letters & Word frequency & Number \\
\hline Early content, low frequency & $2.7(1.9)^{\mathrm{a}}$ & $7.2(2.5)$ & $12.4(9.5)$ & 52 \\
Late content, low frequency & $11.2(3.4)$ & $6.6(2.0)$ & $17.2(40.4)$ & 64 \\
Early content, high frequency & $2.5(0.7)$ & $6.8(2.2)$ & $344.4(429.4)$ & 50 \\
Late content, high frequency & $12.0(3.4)$ & $6.2(2.0)$ & $427.2(497.7)$ & 60 \\
All content (open class) & $7.5(5.2)$ & $6.7(2.2)$ & $200.0(378.1)$ & 226 \\
Function (closed class) & $8.1(4.2)$ & $3.0(1.3)$ & $19,436(22,769)$ & 1429 \\
Final incongruous & $13.6(3.8)$ & $6.5(1.6)$ & $73.7(84.1)$ & 119 \\
Final congruous & $13.1(3.6)$ & $6.3(1.3)$ & $73.5(93.9)$ & 121 \\
\hline
\end{tabular}

a Mean (SD).

Fischl et al., 1999a). First, the cortical white matter was segmented, and the estimated border between gray and white matter was tessellated, providing a topologically correct representation of the surface with $\sim 150,000$ vertices per hemisphere. For the inverse computation, the cortical surface was decimated to approximately 3000 dipoles per hemisphere (i.e., 1 dipole per $\sim 10 \mathrm{~mm}$ of cortical surface). Finally the folded surface tessellation was "inflated," to unfold cortical sulci, thereby providing a convenient format for visualizing cortical activation patterns (Dale and Sereno, 1993; Dale et al., 1999; Fischl et al., 1999a). For purposes of intersubject averaging, the reconstructed surface for each subject was morphed into an average spherical representation, optimally aligning sulcal and gyral features across subjects while minimizing metric distortions (Fischl et al., 1999b). In two subjects, the MR image was of insufficient technical quality to permit accurate reconstruction, and they were not included in the distributed source analysis.

Forward solution. The boundary element method (BEM) was used for calculating the signal expected at each magnetoencephalograph sensor, for each dipole location (deMunck, 1992; Oostendorp and Van Oosterom, 1992). The computation of the MEG forward solution has been shown to require only the inner skull boundary to achieve an accurate solution (Meijs et al., 1988; Hamalainen and Sarvas, 1989).

Inverse solution. To estimate the time courses of cortical activity, the noise-normalized, anatomically constrained linear estimation approach described in Dale et al . (2000) was used. This approach is similar to the generalized least-squares or weighted minimum norm solution (Hamalainen and IImoniemi, 1984; Dale and Sereno, 1993), but the estimate is normalized for noise sensitivity (Dale et al., 2000). The noise normalization has the effect of greatly reducing the variation in the point-spread function between locations (Liu et al., 2002). Of specific interest to this study, simulations using the same sensor array and analysis method as the current study indicated that the point-spread func- tion is typical in the areas where intracranial EEG has identified N400 generators (Liu et al., 2002).

Noise sensitivity-normalized cortical surface-constrained minimum norm inverse solutions were calculated every $5 \mathrm{~ms}$ for every condition and every individual. These movies were then averaged on the cortical surface across individuals and the significance of activation at each site was calculated using an F test (Dale et al., 2000; Dhond et al., 2001). The minimum significance threshold for the displayed activations is shown on each figure and is $P<10^{-5}$, except for the weak effect of word frequency ( $P<0.0005)$. The significance of the comparisons between different conditions at particular locations and latencies is directly indicated by these maps.

In summary, this approach provides dynamic statistical parametric maps (dSPMs) of cortical activity, similar to the statistical maps typically generated using fMRI or PET data, but with a temporal resolution of 5 ms. Even when applied to the same data set, the ECD versus dSPM localizations can be different because they use different aspects of the data to estimate and localize the sources. ECD calculations minimize the least-mean-square difference between a dipolar pattern and the observations in sensor space. The ECD calculations do not take into account the reliability of the measures or the shape of the cortical surface. In contrast, dSPMs do take reliability and shape into account as they estimate the significance of a pattern of activity over the surface of the cortex, i.e., in source space.

Presenting data as statistical maps allows significant responses to be readily visualized in data sets where absolute amplitude is not a reliable indicator of significance due to large differences in variance across locations. This is the case with fMRI as well as MEG. In addition, absolute amplitude estimates of specific cortical locations are problematic with MEG because dipole strength is confounded with generator area. That is, it is often not possible to reliably distinguish a weak but extended generator from one that is strong 
but focal. In addition, dipole strength is decreased to an unknown extent by cancellation between different nearby cortical areas. Finally, there is a complex interaction between depth of the dipoles and amplitude, with deeper dipoles usually being modeled as stronger for technical reasons which may not necessarily reflect neurobiological reality. Thus, it is difficult to compare source amplitudes across sites. However, source amplitudes at a given location can generally be compared across conditions provided that the model remains constant. For many of the dSPMs presented below, the same noise estimate is used when comparing congruous versus incongruous sentence completions, early versus late sentence order, and high versus low word frequency. Since the activity at each cortical location is scaled by the estimated noise at that location, the differences in significance at a given location in the dSPM also indicate differences in estimated source amplitude.

\section{RESULTS}

\section{Fields}

MEG signals with multiple peaks and a complex topography were evoked by both congruous and incongruous words (Fig. 1). To isolate the field pattern associated with the N400 component, the signals evoked by sentence-terminal congruous words were subtracted from those evoked by incongruous words. The resulting fields were large and stable, with a dipolar pattern over the left temporal area in all subjects (Fig. 2).

The results are presented in two main sections describing (1) equivalent current dipole models and (2) distributed source models of the experimental effects. In each section, we first address the manipulation expected to produce the largest N400m effect: congruous versus incongruous sentence completions. We then compare that effect with the three intermediate word contrasts concerning sentence order, word frequency, and lexical class.

\section{Equivalent Current Dipole Modeling}

Final words. A single ECD was modeled to the magnetic field patterns evoked by incongruous sentence-terminal words, after subtracting that evoked by congruous words (Fig. 2). Sources were modeled to the magnetic field evoked by incongruous minus congruous sentence-terminal words at a latency near its maximum of $440 \pm 37 \mathrm{~ms}$ when the field appeared clearest. Fields were modeled separately in each hemisphere, when visible, using $45 \pm 4$ contiguous channels where the signal was greatest. The goodness-of-fit (i.e., the percentage of variance explained, g) was $56 \pm 9 \%$, and the radius of the $95 \%$ confidence volume was estimated as $2.1 \pm 1.2 \mathrm{~mm}$.
When the ECD in each subject was superimposed on that subject's MR image, a consistent localization could be observed, with the ECD lying in or near the superior temporal sulcus, $\sim 3 \mathrm{~cm}$ from the temporal tip in all 10 subjects (Fig. 2). After normalization of the brains (Talairach and Tournoux, 1988), the ECDs were located at the Talairach coordinates: $-86 \pm 6,-2 \pm 6,-3 \pm 12$ (mean $\pm \mathrm{SD}$, distance in $\mathrm{mm}$ to the right of midline, anterior to the anterior commissure, and above the anterior commissure-posterior commissure plane).

The best-fitting ECDs for the sentence congruity effect were then applied to the unsubtracted fields elicited by the congruous and incongruous final words. While keeping the location and direction of each ECD constant, dipole magnitudes were allowed to vary as a function of time, thus producing the best match to the observed field patterns in the "least-mean-squares" sense. In effect, the ECD was used as a spatial filter. J ust as with other filters, unwanted activity, if it is strong and lies within the "bandpass" of the filter, may produce a spurious signal. This is true of many measurement strategies. For example, the N400e is sometimes quantified as its amplitude at the scalp location $\mathrm{Cz}$, referentially recor ded to the mastoid. This measure of the N400e is a kind of spatial filter with a "bandpass" equal to the lead field of that electrode derivation. This is a very large "bandpass" compared with that of the ECDs used in this study. Although spurious activity could have leaked through the filter, qualitatively similar results were obtained with the distributed modeling technique, which makes quite different assumptions (see bel ow), as well as from N400e recordings (see Introduction).

The ECD to incongruous words had a peak latency of $432 \pm 29 \mathrm{~ms}$, with average peak amplitude $27.3 \pm 11.8$ nAm. Since no peak was visible to congruous words, the dipole strength was estimated as the average deviation from baseline from 360 to 450 ms after word onset for each of the 10 subjects. Measured in this way, the average ECD amplitude was $23.3 \pm 11.8 \mathrm{nAm}$ to incongruous sentence endings and $-6.5 \pm 10.0$ nAm to congruous endings (paired t test, $\mathrm{P}<0.001$ ).

Intermediate words. We also examined the three other experimental contrasts previously shown to influence the electrical N400: (1) content words occurring early versus late in their sentences, (2) low- versus high-frequency sentence-intermediate words (although this effect was weak in the present study), and (3) content versus function words. Figure 3 shows contour maps of the evoked magnetic field and the best-fitting equivalent dipoles (at $400 \mathrm{~ms}$ ) for a single subject in whom each dipole was independently derived. The left temporal field elicited by incongruous sentence completions also appeared to be stronger for content words than for function words, and for words occurring early in their sentences after little semantic context as com- 

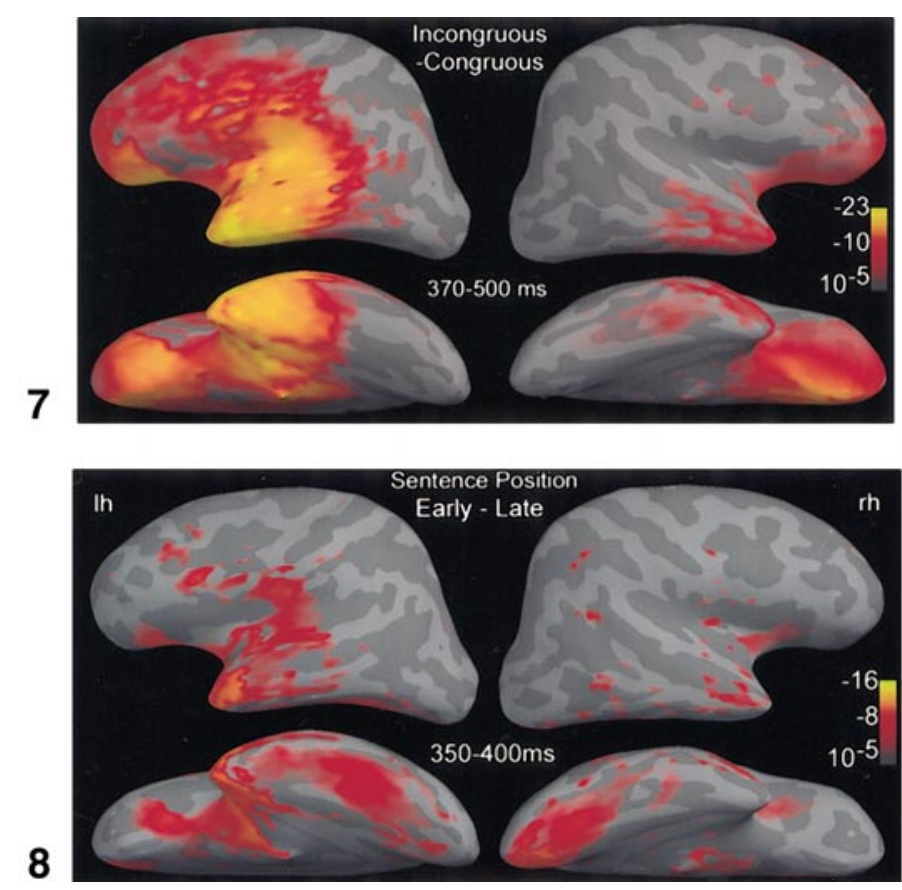

FIG. 7. Average cortical activity patterns in the $\mathbf{N} 400$ latency range to sentence-terminal incongruous minus congruous words. In the left hemisphere, activation is estimated to be widespread in the anterior temporal, perisylvian, orbital, frontopolar, and dorsolateral prefrontal cortices. In the right hemisphere, the orbital, as well as, weakly, the right anterior temporal cortices are activated. Threshold $\mathrm{P}<10^{-5}$; full red $\mathrm{P}<10^{-10}$; full yellow $\mathrm{P}<10^{-23}$.

FIG. 8. Estimated cortical activity patterns to words occurring early versus late in the sentence. Overall activation is left frontotemporal, a pattern similar to but less significant than that observed to incongruous words (Fig. 7). However, in the word order comparison there is additional activation in left ventral occipitotemporal and right ventral occipital cortices. Conversely, there appears to be relatively less activation in the anterior orbital and frontopolar cortices. Threshold $\mathrm{P}<10^{-5}$; full red $\mathrm{P}<10^{-8}$; full yellow $\mathrm{P}<10^{-16}$.

pared with later words. In contrast, the impact of word frequency was weak.

The ECD modeled for the sentence congruity effect was applied to the three intermediate word contrasts to see if the same dipole would yield the expected pattern of early words greater than late, low frequency greater than high, and content greater than function. As above, dipole strength was estimated as the average deviation from baseline from 360 to 450 ms after word onset for all 10 subjects. Figure 4 shows mean dipole strengths. ECD strength was clearly greater for intermediate content as compared with function words $(P<$ 0.001 ). Among the intermediate content words, average ECD strength was greatest for low-frequency words occurring early in the sentence, slightly lower for early high-frequency words, and clearly smaller for late words. ANOVA with factors of word order and word frequency found a main effect of position $(F(1,9)$ $=24.1, \mathrm{P}<0.001)$, but the frequency effect did not reach significance $(F(1,9)=3.3, P<0.10)$ or yield a significant interaction with position $(F(1,9)<1)$. ECD strength was comparable for early content words as compared with sentence-terminal incongruous words.

Effects of baseline. The above values were obtained using a baseline period beginning $50 \mathrm{~ms}$ prior to the word onset and ending $5 \mathrm{~ms}$ after. This baseline could reflect continuing activity from the preceding word. Across most comparison conditions, there is no reason to suspect that the baseline would be different, and thus influence the results. However, some EEG recordings have shown a rising negativity over the course of a sentence (Kutas and Hillyard, 1980), so baseline levels might be different for earlier content words than for later content or sentence-terminal words. Indeed, the baseline did change significantly between the first and terminal words $(0.8 \pm 1.9 \mathrm{nAm}$ vs $-0.9 \pm 1.9 \mathrm{nAm}, \mathrm{P}<$ 0.05). However, no difference was found between early and late content words $(-0.9 \pm 3.4 \mathrm{nAm}$ vs $-0.2 \pm 1.7$ $\mathrm{nAm}, \mathrm{P}>0.2$ ). Because high- and low-frequency words each occurred equally often early and late in the sentence, no differences were expected in their baselines and none were found $(-0.7 \pm 2.2 \mathrm{nAm}$ vs $-0.5 \pm 2.3$ nAm, $P>0.2$ ). Similarly, because congruent and incongruent words each occurred in the sentence-terminal position, no differences were expected in their baselines and none were found $(-0.6 \pm 1.8 \mathrm{nAm}$ vs $-1.1 \pm$ $2.4 \mathrm{nAm}, \mathrm{P}>0.2)$. As noted above, function words are preceded more often by content words than vice versa, and so they may have a baseline that is more contaminated by N400-like activity. Again, however, no differences were found in their baselines $(0.1 \pm 1.4 \mathrm{nAm}$ vs $-0.6 \pm 1.9 \mathrm{nAm}, \mathrm{P}>0.2)$. to further evaluate these possible effects, we analyzed ECD amplitudes without baseline correction. This analysis found estimated ECD amplitude as larger to early than late content words ( $23.0 \pm 13.9$ nAm vs $5.2 \pm 6.7$ nAm, $P<0.001)$, but no significant difference in estimated ECD amplitude to high- versus low-frequency content words (10.9 \pm 11.7 nAm vs $16.2 \pm 15.4 \mathrm{nAm}, \mathrm{P}>0.1)$. Similarly, incongruous words evoked a larger ECD

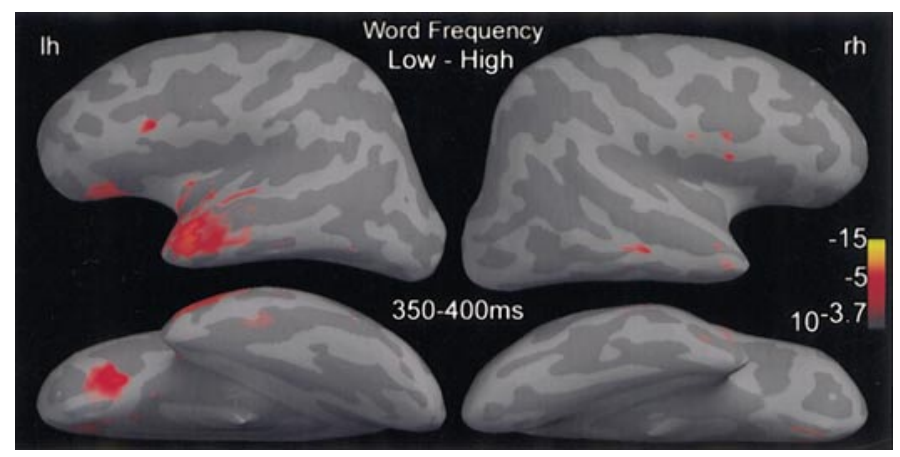

FIG. 9. Estimated cortical activity patterns to high- versus lowfrequency words. Weakly significant activation is present in the left anterolateral temporal and orbitofrontal cortices. Threshold $\mathrm{P}$ 0.0005; full red $\mathrm{P}<10^{-5}$; full yellow $\mathrm{P}<10^{-15}$. 


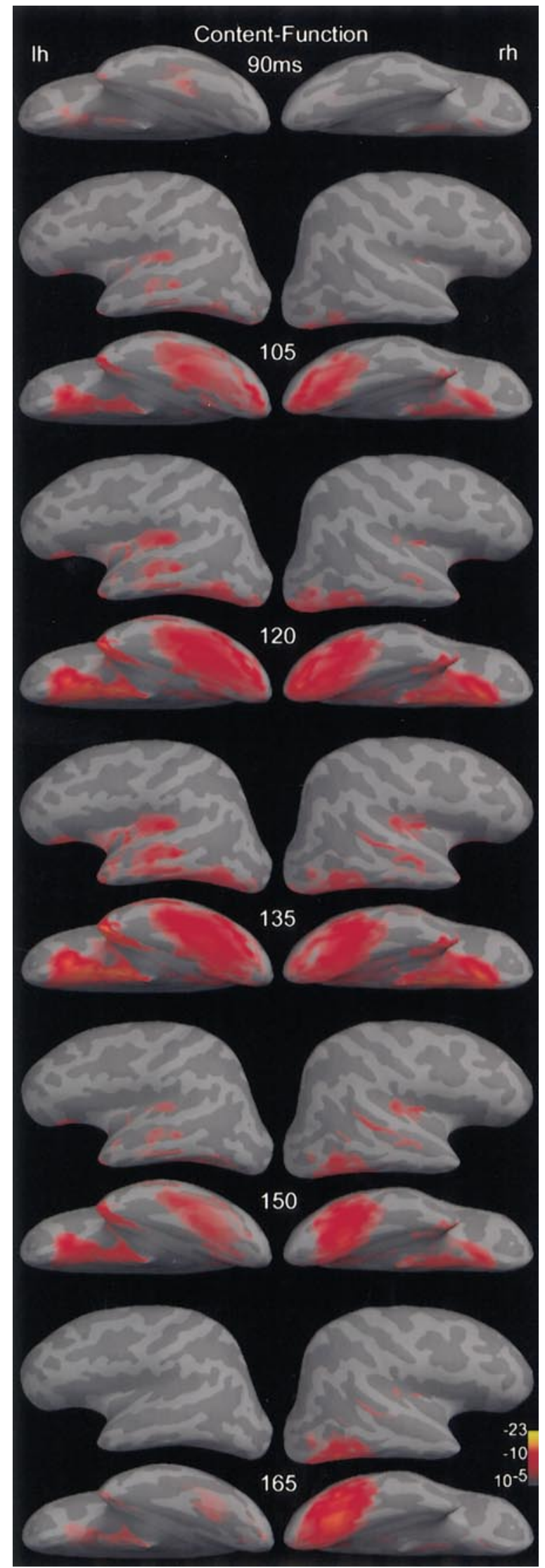

than incongruous $(22.2 \pm 10.6 \mathrm{nAm}$ vs $-7.2 \pm 9.3$ nAm, $\mathrm{P}<0.001)$. Finally, content words evoked a larger ECD than did function (13.6 $\pm 7.6 \mathrm{nAm}$ vs $-1.1 \pm 5.7 \mathrm{nAm}, \mathrm{P}<0.001)$. This pattern of results is identical to that described above, obtained using a baseline immediately preceding the analyzed word.

Peak amplitude and latency measures. The conclusion that sentence position but not word frequency significantly affects $\mathrm{N} 400 \mathrm{~m}$ amplitude was confirmed by peak amplitude measurements when available. The average peak amplitude to low-frequency words could be measured in all 10 subjects for both early (30.6 \pm $16.8 \mathrm{nAm})$ and late $(11.9 \pm 5.6 \mathrm{nAm})$ positions in the sentence $(P<0.001)$. A clear peak to early high-frequency words was visible in only 8 subjects. Considering the same 8 subjects, the early words did not produce significantly different amplitude peaks when evoked by low $(27.9 \pm 11.3 \mathrm{nAm})$ - as compared with high $(25.6 \pm 9.9 \mathrm{nAm})$-frequency words $(\mathrm{P}>0.3)$. A peak to late high-frequency content words could be reliably identified in only one subject.

The latency to peak amplitude of the estimated ECD strength curves was shorter for the intermediate words (386 $\pm 40 \mathrm{~ms}$ for the early low-frequency content words, and $374 \pm 28 \mathrm{~ms}$ for the late low-frequency content words), as compared with the sentence-terminal incongruous words ( $432 \pm 29 \mathrm{~ms})$. The early versus late low-frequency words do not differ significantly in latency $(P>0.2)$, but both are significantly shorter than for the incongruous words (both $\mathrm{P}<0.001$ ). Considering only the 8 subjects with a clear peak to early high-frequency words, the early words did not produce significantly different latency peaks when evoked by low (378 $\pm 41 \mathrm{~ms})$ - as compared with high (379 \pm 29 ms)-frequency words. An early visual dipole at $\sim 110$ ms did not change across the above conditions, except that it was smaller to the shorter function words, confirming that simple visual intensity was balanced between conditions.

\section{Dynamic Statistical Parametric Maps}

Distributed inverse solutions were estimated every 5 ms for each cortical dipole, condition, and subject. The dSPM for the difference waveforms between sentenceterminal incongruous minus congruous words indicated a single major effect: activation in the left anterior temporal and posteroventral prefrontal cortices,

FIG. 10. Estimated cortical activity patterns to content versus function words. Differential activation is most prominent in ventral occipitotemporal and orbitofrontal cortices, where it begins in the left at $\sim 90 \mathrm{~ms}$, is strong and bilateral from $\sim 105$ to $135 \mathrm{~ms}$, and becomes progressively focal in the right occipitotemporal cortex at $\sim 150-165$ ms. Activation is also present in the temporal pole and posterosuperior temporal lobe from $\sim 105$ to $150 \mathrm{~ms}$, especially on the left. Threshold $\mathrm{P}<10^{-5}$; full red $\mathrm{P}<10^{-10}$; full yellow $\mathrm{P}<10^{-23}$. 
beginning at $\sim 250 \mathrm{~ms}$ and peaking at $\sim 400 \mathrm{~ms}$ after word onset. The maximum significance for each dipole over the range 300-500 ms in a representative subject is shown in Fig. 5.

Final words. The dSPMs were combined across the 8 subjects with adequate MR images by morphing their brains based on their individual sucal-gyral patterns. The resulting group dSPM to incongruous minus congruous words is shown for five latency ranges in Fig. 6 and 7. During the peak latency range for the scalprecorded N400 (370-500 ms), left anterior temporal and posteroventral prefrontal activation predominated (Fig. 7). However, prior to $370 \mathrm{~ms}$, the pattern of activity evoked by incongruous sentence-terminal words shows a general progression of activation from posterior to anterior sites and from left to right (Fig. 6). Differential activation to incongruous words began in the left planum temporale (Wernicke's area) at $\sim 250$ ms after word onset. After $\sim 20 \mathrm{~ms}$, activity spread to the left superior and inferior temporal sulci, anteroventral temporal lobe (temporal pole and rhinal sulcus), insula, and collateral sulcus at the occipitotemporal junction. Following $\sim 300 \mathrm{~ms}$ the prefrontal cortex became increasingly involved, first in its orbital aspect (gyrus rectus), then in Broca's area (inferior frontal gyrus), and finally, after $\sim 370 \mathrm{~ms}$, in the dorsolateral prefrontal and frontopolar cortices (superior and middle frontal gyri). The right hemisphere did not show activation until $\sim 370 \mathrm{~ms}$, when it was most prominent in orbital cortex, with less significant activations in anterior temporal cortex (Fig. 7).

Intermediate words. Magnetoencephalographic activity evoked by words occurring prior to sentence termination ("intermediate words") was also examined for differences related to presentation order within the sentence (early vs late), word frequency (high vs low), and lexical class (content vs function). Overall activation to words occurring early versus late in the sentence (Fig. 8) is left frontotemporal, a pattern similar to but less significant than that observed to incongruous versus congruous sentence-terminal words (compare to Fig. 7). However, in the sentence position comparison there is additional activation in the right ventral occipital cortex, as well as relatively more activation in the left ventral occipitotemporal cortex. Conversely, there appears to be relatively less activation in the anterior orbital and frontopolar cortices.

Compared with sentence position and congruity effects, word frequency had only a weakly significant effect on the MEG signal. However, the generators of the difference between signals evoked by high- versus low-frequency words were again estimated to be located in left anterolateral temporal and orbitofrontal cortices (Fig. 9). No significant differences prior to 350 ms were observed in the signals evoked by early versus late or high- versus low-frequency words.
A distributed solution was also calculated for the difference between the MEG signals evoked by the intermediate content words chosen for the above analyses of sentence position and word frequency, in comparison to intermediate function words. In this comparison, it is important to bear in mind that the function words had significantly fewer letters and higher word frequency, as compared with the content words. This comparison revealed strong differential activation beginning as early as $90 \mathrm{~ms}$ after word onset, and estimated to arise in left ventral occipitotemporal and oribitofrontal locations (Fig. 10). Differential activation became rapidly bilateral in the same locations, and also involve cortex in the temporal pole and posterosuperior temporal lobe from 105 to $150 \mathrm{~ms}$. Although the latter areas were more strongly activated on the left at most latencies, activation became progressively focal in the right occipitotemporal cortex from $\sim 150$ to $165 \mathrm{~ms}$. Differential activation was then not seen until after $400 \mathrm{~ms}$, when it might be related to the subsequent word and so is not presented here.

\section{DISCUSSION}

The current study has implications for both the spatiotemporal dynamics of sentence comprehension and the methods best suited to study those dynamics. A strong MEG signal was observed by contrasting the same conditions that have previously been found to result in the scalp N400e potential. The MEG signal has a latency similar to that of the N400e, and thus is termed the $\mathrm{N} 400 \mathrm{~m}$.

\section{Sentence-Terminal Words}

I nitial analysis of the N400m used the most common method, fitting of an ECD that best matches the observed field pattern. The resulting N400m ECD has a reasonable localization, strongly lateralized to the left hemisphere, in or near the superior temporal sulcus, as has been found in previous studies using similar techniques (Simos et al., 1997; Helenius et al., 1999).

The current study analyzed the same data using a distributed source modeling technique that estimated activity for every cortical location at each time point (Dale and Sereno, 1993). Activity is normalized at each location by its estimated noise, resulting in dynamic statistical parametric maps (Dale et al., 2000). While uncertainties associated with the inverse solution limit the accuracy of these results (Dale and Halgren, 2001), simulation studies indicate that large errors are highly unlikely (Dale et al., 2000; Liu et al., 2002). The distributed solution was consistent with the ECD solution, in that it included the left superior temporal area where the ECDs were located. However, the distributed solution also included anteroventral temporal, orbitofrontal, and posteroventral prefrontal cortices in 
the left hemisphere. The distributed solution also included orbitofrontal and (weakly) anterior temporal cortices in the right hemisphere. These locations match those that have been demonstrated to generate potentials at this latency in the same or similar paradigms (see Introduction). In particular, although words in an explicit recognition paradigm do evoke $\mathrm{N} 400 \mathrm{e}-$ like activity in the region of the left superior temporal sulcus (Halgren et al., 1994a; Guillem et al., 1995; Elger et al., 1997), the most prominent generators are in the anteroventral temporal (Smith et al., 1986; Halgren et al., 1994a; McCarthy et al., 1995; Fernandez et al., 1998; Grunwald et al., 1998), orbitofrontal, and posteroventral prefrontal (Halgren et al., 1994b; Guillem et al., 1999) cortices. Thus, the distributed source modeling technique used here identifies a distributed network of N400 generators similar to that previously demonstrated with intracranial recordings.

An additional similarity between the areas identified by distributed source modeling here and previous intracranial recordings is that both find bilateral N400 generators in ventroposterior prefrontal and anteroventral temporal cortices (Smith et al., 1986; Halgren et al., 1994a,b; Klopp et al., 1999). Although there are some indications from intracranial recordings that the N400 generators are larger on the left to words (Smith et al., 1986; Marinkovic et al., 2000), the confounding influences of pathology and idiosyncratic electrode placement render interhemispheric comparisons problematic. The current results in normal subjects provide converging evidence that the $\mathrm{N} 400 \mathrm{~m}$ evoked by words is due to bilateral generators, more prominent in the left hemisphere. Similar results have been obtained in previous studies using the same distributed anatomically constrained noise-normalized source modeling technique, on MEG fields evoked by single words (Dale et al., 2000) or word stems (Dhond et al., 2001). In a group of seven subjects, Helenius et al. (1999) were able to model more ECDs sensitive to incongruous sentence endings in the left than in the right hemisphere (15 vs 5). Semantically anomal ous sentences have also been found with $\mathrm{fMRI}$ to produce distributed, left greater than right but bilateral cortical activation (Kuperberg et al., 2000). It would be interesting to determine if this degree of lateralization is also present in female subjects, who may have reduced functional lateralization in verbal paradigms, as well as reduced anatomical asymmetries in related areas (Wegesin, 1998; Shapleske et al., 1999; Kansaku and Kitazawa, 2001).

In conclusion, the ECD localizing near the left superior temporal sulcus has many of the cognitive characteristics previously described for the N400 potential, but the localization is not entirely consistent with fMRI and IEEG data. These results emphasize the importance of recalling that the ECD is an equivalent dipole that in this case represents an extended discontinuous generator in multiple structures. The distributed solution seems more appropriate for this type of very distributed generator, judging by its better correspondence with iEEG and fMRI. However, even with distributed generators, the ECD is useful in allowing the generator to be quantified across conditions and time, in a manner that may be less prone to noise than estimates of individual cortical dipoles in a distributed solution. Of course, this advantage of the ECD is subject to the assumption that the spatial distribution of the generator is constant across conditions and time, an assumption that is unlikely to be completely accurate.

Although the $\mathrm{N} 400$ peaks at $\sim 400 \mathrm{~ms}$, previous studies have consistently found that the differential response to visual sentence-terminal incongruous words begins at $\sim 250 \mathrm{~ms}$ (Halgren, 1990; Helenius et al., 1999). The current results confirmed this finding, and in addition found that the source distribution changed dramatically from the first appearance of the differential response to its maximal expression. Distributed source modeling located the differential activation from 250 to $265 \mathrm{~ms}$ almost exclusively to the planum temporale. By $\sim 270 \mathrm{~ms}$, differential activation spread to the left anteroventral temporal lobe, then by $\sim 300$ ms to left prefrontal cortex. These results are in contrast to the distributed solutions obtained for N400mlike activity evoked by visually presented words or word stems in previous studies. For example, the earliest differential response was estimated to lie in anteromedial temporal sites when words were repeated in an explicit memory paradigm (Dale et al., 2000) and in posteroventral sites when word stems were repeated in a stem completion paradigm (Dhond et al., 2001). In all cases, the differential response in the left ventral frontotemporal circuit begins only after all components of the circuit have been active for $\sim 50$ to $100 \mathrm{~ms}$. However, the ultimate circuitry involved at $\sim 350$ to 450 ms appears to be very similar. The possibility that the left anterior temporal lobe performs essential early processing during the $\mathrm{N} 400$ is consistent with the effects of brain lesions on the scalp N400 (Kutas et al., 1988a; Smith and Halgren, 1989).

These data generally support a model wherein potentially meaningful stimuli activate a semantic contextual integration process distributed across cortical areas specialized for different aspects of this process (Halgren, 1990). Specifically, they suggest that visually presented words evoke an N400 process that engages a distributed frontotemporal network, beginning at $\sim 200 \mathrm{~ms}$. This network is modulated by various factors that influence the difficulty of integrating the word with the current cognitive context. The integration takes place in a network, with different areas contributing more to different aspects of the integration, so that when integration is eased by repetition priming, medial temporal sites may be the first to show 
differential activity. Conversely, when integration is eased by contextual semantic congruity, the current results suggest that Wernicke's area may be the first to show facilitated processing. Eventually, multiple areas all come to show differential processing which presumably results in the widespread differential MEG response.

\section{Sentencel ntermediate Words}

As reviewed in the Introduction, previous scalp EEG recordings have suggested that widespread scal p negativities with peak latency $\sim 400 \mathrm{~ms}$ can be observed not only when contrasting sentence-terminal incongruous versus congruous words, but also when contrasting various classes of sentence-intermediate words (Kutas and Van Petten, 1994; Van Petten, 1995; Kutas and Federmeier, 2000). The scalp topography of the N400s recorded in these different situations are similar, suggesting that they may reflect similar neuronal processes. However, the observed scalp topographies are also consistent with a variety of different intracranial generators. Stronger conclusions can be drawn from intracranial studies, which, however, are limited by considerations of sampling and pathology.

In the current study, high-density MEG was used to provide a complementary test of this hypothesis in normal subjects. Using again the same distributed source model, very similar patterns of cortical activation were found to be evoked by words with early versus late sentence position, as compared with those evoked by incongruous versus congruous sentence-terminal words. The inference that similar neuronal processes are engaged by sentence-intermediate versus sentence-terminal words was further supported by the ECD analysis of the same data, where the ECD modeled on the sentence-terminal incongruous/congruous contrast was found to be differentially activated to intermediate words with early as opposed to late sentence position. The cortical activation to sentence-intermediate words with low (as compared with high) lexical frequency was also mapped with the distributed model to a left frontotemporal network similar to that observed to the sentence position and congruity contrasts. However, the activation was less widespread and less significant, and the ECD model did not find significant differences associated with word frequency.

These data are consistent with those of previous studies of the N400e cited in the Introduction (Kutas and Van Petten, 1994; Van Petten, 1995; Kutas and Federmeier, 2000). The current data extended those studies because MEG is maximally sensitive to different cortical areas than EEG, and thus the N400m's replication of the cognitive profile of the N400e suggests that this is a robust property of the underlying neuronal activity. Furthermore, compared with the previous N400e studies, the current study used more formal source modeling, based on a larger number of sensors, to compare the response to sentence-terminal versus intermediate words. Thus, these data strongly support the similarity of the neuronal processes that are used to process early content words versus sentence-terminal incongruous words.

At a functional level, these data are consistent with models wherein the N400 to words embodies their semantic integration within the developing cognitive context (Halgren, 1990; Kutas and Federmeier, 2000). As a sentence is read, a cognitive context is constructed that allows the meaning of each successive word to be interpreted. When the word matches the established context, that process is greatly facilitated, as compared with when it does not. Thus, the differential activation to both sentence-intermediate content words and to sentence-terminal incongruous words appears to reflect the same process of cognitive contextual integration.

These results were obtained including only content words in the analyses. When the ECD derived from sentence-terminal incongruous minus congruous words was used to probe the response to sentenceintermediate function words, no activation could be detected. In contrast, pronounced differences were found between sentence-intermediate content and function words in the distributed maps, beginning at very early latencies. At $\sim 120$ to $135 \mathrm{~ms}$, these differences are pronounced in ventral occipitotemporal and orbitofrontal cortices. These intriguing results suggest that very early processes may select content words for cognitive integration (using the N400 process as defined above), while relegating function words to a distinct syntactic processing route. This conclusion must remain speculative given that the content and function words differed in their length and lexical frequency.

\section{CONCLUSIONS}

Brain activity that is generated in multiple extended locations (as indicated by depth recordings) may be more realistically modeled using anatomically constrained distributed sources, as compared with the standard equivalent current dipoles. A widespread, mainly left, frontotemporal process peaking at $\sim 400$ ms is stronger when contextual semantic processing is more difficult. The dynamics of this process to sentence-terminal incongruous versus congruous words suggests that it arises in Wernicke's area, spreads first to the anteroventral temporal lobe, and finally to Broca's area. Similar areas are involved in the contextual semantic processing of words occurring earlier in the sentence.

\section{ACKNOWLEDGMENTS}

This work was supported by the USPHS (NIH Grant NS18741), the National Foundation for Functional Brain Imaging (DOE Grant 
DE-FG03-99E R62764), and the Human Frontiers Science Program Organization (Grant RG25/96). We thank Seppo Ahlfors, Kim Paulson, Bruce Fischl, Arthur Liu, Valerie Carr, Thomas Witzel, Robert $\mathrm{J}$. Thoma, Bruce Rosen, and William Orrison. These data were collected at the Center for Advanced Medical Technology, Department of Radiology, University of Utah, Salt Lake City.

\section{REFERENCES}

Ahonen, A. I., Hämäläinen, M. S., Kajola, M. J ., Knuutila, J . E. T., Laine, P. P., Lounasmaa, O. V., Parkonen, L. T., Simola, J . T., and Tesche, C. D. 1993. 122-channel SQUID instrument for investigating the magnetic signals from the human brain. Phys. Scr. T 49: 198-205.

Curran, T., Tucker, D. M., Kutas, M., and Posner, M. I. 1993. Topography of the N400: Brain electrical activity reflecting semantic expectancy. Electroencephalogr. Clin. Neurophysiol. 88: 188-209.

Dale, A. M., and Halgren, E. 2001. Spatiotemporal mapping of brain activity by integration of multiple imaging modalities. Curr. Opin. Neurobiol. 11: 202-208.

Dale, A. M., and Sereno, M. I. 1993. I mproved localization of cortical activity by combining EEG and MEG with MRI cortical surface reconstruction: A linear approach. J . Cogn. Neurosci. 5: 162-176.

Dale, A. M., Fischl, B., and Sereno, M. I. 1999. Cortical surface-based analysis. I. Segmentation and surface reconstruction. Neurol mage 9: 179-194.

Dale, A. M., Liu, A. K., Fischl, B. R., Buckner, R. L., Belliveau, J . W., Lewine, J . D., and Halgren, E. 2000. Dynamic statistical parametric mapping: Combining $\mathrm{fMRI}$ and $\mathrm{MEG}$ for high-resolution imaging of cortical activity. Neuron 26: 55- 67.

deMunck, J . C. 1992. A linear discretization of the volume conductor boundary integral equation using analytically integrated elements. IEEE Trans. Biomed. Eng. 39: 986-990.

Dhond, R. P., Buckner, R. L., Dale, A. M., Marinkovic, K. M., and Halgren, E. 2001. Spatiotemporal maps of brain activity underlying word generation and their modification during repetition priming. J. Neurosci. 21: 3564-3571.

Elger, C. E., Grunwald, T., Lehnertz, K., Kutas, M., Helmstaedter, C., Brockhaus, A., Van Roost, D., and Heinze, H. J . 1997. Human temporal lobe potentials in verbal learning and memory processes. Neuropsychologia 35: 657-667.

Fernandez, G., Weyerts, H., Tendolkar, I., Smid, H. G., Scholz, M., and Heinze, H.J . 1998. Event-related potentials of verbal encoding into episodic memory: Dissociation between the effects of subsequent memory performance and distinctiveness. Psychophysiology 35: 709-720.

Fischl, B., Sereno, M. I., and Dale, A. M. 1999a. Cortical surfacebased analysis. II. Inflation, flattening, a surface-based coordinate system. Neurol mage 9: 195-207.

Fischl, B., Sereno, M. I., Tootell, R. B., and Dale, A. M. 1999 b. $\mathrm{High}$-resolution intersubject averaging and a coordinate system for the cortical surface. Hum. Brain Mapp. 8: 272-284.

Friederici, A. D., Hahne, A., and von Cramon, D. Y. 1998. First-pass versus second-pass parsing processes in a Wernicke's and a Broca's aphasic: Electrophysiological evidence for a double dissociation. Brain Lang. 62: 311-341.

Ganis, G., Kutas, M., and Sereno, M. I. 1996. The search for "common sense": An electrophysiological study of the comprehension of words and pictures in reading. J . Cogn. Neurosci. 8: 89-106.

Grunwald, T., Lehnertz, K., Helmstaedter, C., Kutas, M., Pezer, N., Kurthen, M., Van Roost, D., and Elger, C. E. 1998. Limbic ERPs predict verbal memory after left-sided hippocampectomy. NeuroReport 9: 3375-3378.

Guillem, F., N'Kaoua, B., Rougier, A., and Claverie, B. 1995. Intracranial topography of event-related potentials (N400/P600) elicited during a continuous recognition memory task. Psychophysiology 32: 382-392.

Guillem, F., Rougier, A., and Claverie, B. 1999. Short- and long-delay intracranial ERP repetition effects dissociate memory systems in the human brain. J . Cogn. Neurosci. 11: 437- 458.

Haan, H., Streb, J ., Bien, S., and Rosler, F. 2000. Individual cortical current density reconstructions of the semantic N400 effect: Using a generalized minimum norm model with different constraints (L1 and L2 norm). Hum. Brain Mapp. 11: 178-192.

Hagoort, P., Brown, C. M., and Swaab, T. Y. 1996. Lexical-semantic event-related potential effects in patients with left hemisphere lesions and aphasia, and patients with right hemisphere lesions without aphasia. Brain 119: 627-649.

Halgren, E. 1990. Insights from evoked potentials into the neuropsychological mechanisms of reading. In Neurobiology of Cognition (A. Scheibel and A. Weschsler, Eds.), pp. 103-150. Guilford, New York.

Halgren, E., Baudena, P., Heit, G., Clarke, J . M., and Marinkovic, K. 1994a. Spatio-temporal stages in face and word processing. 1. Depth-recorded potentials in the human occipital, temporal and parietal lobes. J. Physiol. (Paris) 88: 1-50.

Halgren, E., Baudena, P., Heit, G., Clarke, J . M., Marinkovic, K., and Chauvel, P. 1994b. Spatio-temporal stages in face and word processing. 2. Depth-recorded potentials in the human frontal and Rolandic cortices. J . Physiol. (Paris) 88: 51- 80.

Hamalainen, M. S., and IImoniemi, R. J . 1984. Interpreting Measured Magnetic Fields of the Brain: Estimates of Current Distributions. Helsinki Univ. of Technology, Helsinki.

Hamalainen, M. S., and Sarvas, J . 1989. Realistic conductivity geometry model of the human head for interpretation of neuromagnetic data. IEEE Trans. Biomed. Eng. 36: 165-171.

Hamalainen, M., Hari, R., Ilmoniemi, R. J ., Knuutila, J ., and Lounasmaa, O. V. 1993. Magnetoencephalography: Theory, instrumentation, and application to noninvasive studies of the working human brain. Rev. Mod. Phys. 65: 413- 497.

Helenius, P., Salmelin, R., Service, E., and Connolly, J. F. 1999. Semantic cortical activation in dyslexic readers. J . Cogn. Neurosci. 11: 535-550.

Helmstaedter, C., Grunwald, T., Lehnertz, K., Gleissner, U., and Elger, C. E. 1997. Differential involvement of left temporolateral and temporomesial structures in verbal declarative learning and memory: Evidence from temporal lobe epilepsy. Brain Cogn. 35: 110-131.

Holcomb, P. J ., and McPherson, W. B. 1994. Event-related brain potentials reflect semantic priming in an object decision task. Brain Cogn. 24: 259-276.

Hunt, S. M. J . 1994. MacProbe: A Macintosh-based experimenter's workstation for the cognitive sciences. Behav. Res. Methods Instrum. Comput. 26: 345-351.

Kansaku, K., and Kitazawa, S. 2001. Imaging studies on sex differences in the lateralization of language. Neurosci. Res. 41: 333-337.

King, J . W., and Kutas, M. 1998. Neural plasticity in the dynamics of human visual word recognition. Neurosci. Lett. 244: 61-64.

Klopp, J. C., Halgren, E., Marinkovic, K., and Nenov, V. I. 1999. Face-selective event-related spectral changes in the human fusiform gyrus. Clin. Neurophysiol. 110: 677-683.

Kucera, H., and Francis, W. N. 1967. Computational Analysis of Present-Day American English. Brown Univ. Press, Providence, RI.

Kuperberg, G. R., McGuire, P. K., Bullmore, E. T., Brammer, M. J ., Rabe-Hesketh, S., Wright, I. C., Lythgoe, D. J., Williams, S. C., and David, A. S. 2000. Common and distinct neural substrates for pragmatic, semantic, and syntactic processing of spoken sentences: An fMRI study. J . Cogn. Neurosci. 12: 321-341. 
Kutas, M., and Federmeier, K. D. 2000. Electrophysiology reveals semantic memory use in language comprehension. Trends Cogn. Sci. 4: 463- 470.

Kutas, M., and Hillyard, S. A. 1980. Reading senseless sentences: Brain potentials reflect semantic incongruity. Science 207: 203205.

Kutas, M., and Hillyard, S. A. 1982. The lateral distribution of event-related potentials during sentence processing. Neuropsychologia 20: 579-590.

Kutas, M., and Hillyard, S. A. 1983. Event-related brain potentials to grammatical errors and semantic anomalies. Mem. Cogn. 11: 539550.

Kutas, M., and Van Petten, C. 1994. Psycholingusistics electrified: Event-related brain potential investigations. In Handbook of Psycholinguistics (M. Gernsbacher, Ed.), pp. 83-143. Academic Press, New York.

Kutas, M., Hillyard, S. A., and Gazzaniga, M. S. 1988a. Processing of semantic anomaly by right and left hemispheres of commissurotomy patients: Evidence from event-related brain potentials. Brain 111: 553-576.

Kutas, M., Van Petten, C., and Besson, M. 1988b. Event-related potential asymmetries during the reading of sentences. Electroencephalogr. Clin. Neurophysiol. 69: 218-233.

Liu, A. K., Dale, A. M., and Belliveau, J. W. 2002. Monte Carlo simulation studies of EEG and MEG localization accuracy. Hum. Brain Mapp. 16: 47-62.

Marinkovic, K., Trebon, P., Chauvel, P., and Halgren, E. 2000. Localized face-processing by the human prefrontal cortex. 2. Faceselective intracerebral potentials and post-lesion deficits. Cogn. Neuropsychol. 17: 187-199.

McCarthy, G., Nobre, A. C., Bentin, S., and Spencer, D. D. 1995. Language-related field potentials in the anterior-medial temporal lobe: I. Intracranial distribution and neural generators. J . Neurosci. 15: 1080-1089.

Meijs, J. W., Peters, M. J ., Boom, H. B., and Lopes da Silva, F. H. 1988. Relative influence of model assumptions and measurement procedures in the analysis of the MEG. Med. Biol. Eng. Comput. 26: $136-142$.

Munte, T. F., Wieringa, B. M., Weyerts, H., Szentkuti, A., Matzke, M., and J ohannes, S. 2001. Differences in brain potentials to open and closed class words: Class and frequency effects. Neuropsychologia 39: 91-102.

Neville, H. J ., Coffey, S. A., Lawson, D. S., Fischer, A., Emmorey, K., and Bellugi, U. 1997. Neural systems mediating American sign language: Effects of sensory experience and age of acquisition. Brain Lang. 57: 285-308.

Nobre, A. C., and McCarthy, G. 1995. Language-related field potentials in the anterior-medial temporal lobe. II. Effects of word type and semantic priming. J . Neurosci. 15: 1090-1098.

Nobre, A. C., Allison, T., and McCarthy, G. 1994. Word recognition in the human inferior temporal lobe. Nature 372: 260-263.

Olichney, J. M., Van Petten, C., Paller, K. A., Salmon, D. P., I ragui, V. J ., and Kutas, M. 2000. Word repetition in amnesia: Electro- physiological measures of impaired and spared memory. Brain 123: 1948-1963.

Oostendorp, T. F., and Van Oosterom, A. 1992. Source parameter estimation using realistic geometry in bioelectricity and biomagnetism. In Biomagnetic Localization and 3D Modeling $(\mathrm{J}$. Nenonen, H. M. Rajala, and T. Katila, Eds.), Rep. TKK-F-A689. Helsinky Univ. of Technology, Helsinki.

Plante, E., Petten, C. V., and Senkfor, A. J . 2000. Electrophysiological dissociation between verbal and nonverbal semantic processing in learning disabled adults. Neuropsychologia 38: 1669-1684.

Puce, A., Andrewes, D. G., Berkovic, S. F., and Bladin, P. F. 1991. Visual recognition memory: Neurophysiological evidence for the role of temporal white matter in man. Brain 114: 1647-1666.

Shapleske, J ., Rossell, S. L., Woodruff, P. W., and David, A. S. 1999. The planum temporale: A systematic, quantitative review of its structural, functional and clinical significance. Brain Res. Brain Res. Rev. 29: 26- 49.

Simos, P. G., Basile, L. F., and Papanicolaou, A. C. 1997. Source localization of the $\mathrm{N} 400$ response in a sentence-reading paradigm using evoked magnetic fields and magnetic resonance imaging. Brain Res. 762: 29-39.

Smith, M. E., and Halgren, E. 1987. Event-related potentials during lexical decision: Effects of repetition, word frequency, pronounceability, and concreteness. Electroencephalogr. Clin. Neurophysiol. Suppl. 40: 417-421.

Smith, M. E., and Halgren, E. 1989. Dissociation of recognition memory components following temporal lobe lesions. J . Exp. Psychol. Learn. Mem. Cogn. 15: 50-60.

Smith, M. E., Stapleton, J . M., and Halgren, E. 1986. Human medial temporal lobe potentials evoked in memory and language tasks. Electroencephalogr. Clin. Neurophysiol. 63: 145-159.

Talairach, J ., and Tournoux, P. 1988. Co-planar Stereotaxic Atlas of the Human Brain. Thieme, New York.

Van Petten, C. 1995. Words and sentences: Event-related brain potential measures. Psychophysiology 32: 511-525.

Van Petten, C., and Kutas, M. 1990. Interactions between sentence context and word frequency in event-related brain potentials. Mem. Cogn. 18: 380-393.

Van Petten, C., and Kutas, M. 1991. Influences of semantic and syntactic context on open- and closed-class words. Mem. Cogn. 19: 95-112.

Van Petten, C., and Rheinfelder, H. 1995. Conceptual relationships between spoken words and environmental sounds: Event-related brain potential measures. Neuropsychologia 33: 485-508.

Van Petten, C., and Senkfor, A. J . 1996. Memory for words and novel visual patterns: Repetition, recognition, and encoding effects in the event-related brain potential. Psychophysiology 33: 491-506.

Van Petten, C., Coulson, S., Rubin, S., Plante, E., and Parks, M. 1999. Time course of word identification and semantic integration in spoken language. J . Exp. Psychol. Learn. Mem. Cogn. 25: 394417.

Wegesin, D. J . 1998. Event-related potentials in homosexual and heterosexual men and women: Sex-dimorphic patterns in verbal asymmetries and mental rotation. Brain Cogn. 36: 73-92. 